\title{
Human Resources Availability in the Czech Health Care System
}

\author{
L. O. Dittrich • D. Stara
}

Published online: 15 October 2014

(C) International Atlantic Economic Society 2014

\section{JEL Category I00}

This paper suggests that the problem with the availability of human resources in the Czech health care system is not the absolute shortage of physicians, as is frequently presented, but in the professional aging of physicians and its impact on the quality of delivered care. The data from the Czech Institute of Health Care Information and Statistics actually show a slight but steady increase in the number of physicians during the 1990 to 2012 period. The data also show a substantial professional aging of physicians in most clinical specialties. We use existing studies [Choudry et al., Annals of Internal Medicine, 2005] of the relationship between the length of clinical experience and quality of health care, to arrive at our conclusion.

A significant body of literature dealing with the quality of medical care delivered by physicians reports an inverse correlation between the length of time in practice and outcomes such as up to date knowledge, diagnostic skills, adherence to the standards of appropriate therapy and worse health care results. In other words, physicians who have been in practice too long may be at risk of providing lower quality health care. Choudry et al. 2005 found after a thorough review of existing studies of the relationship between the age of physicians and quality of care that "of 62 published studies more than half suggested that physician performance declined over time for all outcomes measured. Only one study showed improved performance for all outcomes measured".

We examined changes in physician age profiles for a wide spectrum of clinical specialties. The data show a dramatic shift in the age profile pyramid for most specialties between 1992 and 2012. In 1992, in 49 specialties, the ratio of physicians younger than 50 years to physicians older than 50 years was 2 or more. In four specialties the ratio was less than 2 . Only in two specialties was the ratio is less than 1 , indicating a higher proportion of older physicians in the specialties.

L. O. Dittrich $(\bowtie) \cdot$ D. Stara

Czech University of Life Sciences Prague, Prague, Czech Republic

e-mail: dittrich@pef.czu.cz 
In 2012 we see a massive reversal of ratios. In 2012 for only nine specialties is the ratio of physicians younger than 50 to physicians older than 50 years 2 or more. In 30 specialties the ratio is substantially less than 2 . In 15 specialties the ratio is less than 1.

The majority of the above studies involve general practitioners, pediatricians, internists and surgeons. In the Czech Republic the ratio of general practitioners younger than 50 to older than 50 was 2.3 in 1992. In 2012 the ratio was 0.42 . Studies oriented towards the knowledge of general practitioners report an inverse relationship between the age of the physician and his or her test score in different areas. The ratio of pediatricians younger than 50 to older than 50 was 3.7 in 1992. In 2012 the ratio had dropped to 0.28 . Reviewed studies show that pediatricians who graduated a longer time ago are less likely to adhere to the guidelines for treatment of fever in infants. The ratio of internists younger than 50 to older than 50 was 3.9 in 1992. In 2012 the ratio was 1.8. Most studies found a significant inverse correlation between general medical knowledge of internists and lengths of time since certification. The ratio of surgeons younger than 50 to older than 50 was 4.1 in 1992. In 2012 the ratio was 1.2. Reviewed studies found a significant negative correlation between general surgical knowledge and the age of tested surgeons and also show that a higher number of years in practice is associated with higher mortality ratios after coronary bypass surgery. The explanation for finding that the age profile has shifted markedly toward older physicians may be the following: 1. Physicians are delaying their retirements. 2. Following the 1989 regime change, a number of physicians entered non-medical professions and after working some time outside of the medical profession, they returned. 3. Young physicians are increasingly migrating abroad.

Choudry et al., Annals of Internal Medicine, 2005 reason that the "professionally" older physicians tend to underperform because:

“Older physicians' approaches are created during training and not updated regularly. A rapid growth in practice innovations represents fundamental shift away from procedures and techniques consistent with the physician's preexisting knowledge. It may also reflect substantial environmental changes in the medicine over the past several decades such as acceptance of evidence based medicine and introduction of quality assurance techniques, such as disease management."

We conclude that a system of quality improvement interventions should be considered in order to deal with the problem. An efficacy verifiable system of continuing education can be suggested as an obvious intervention strategy. This should include academic detailing programs, as well as traditional in-class continuing education. The authors are currently working with insurance companies in order to come up with an estimate of cost due to the delay in the recognition and treatment of some diseases. Hypertension and diabetes are the first disease conditions we plan to look at. 